Pacific Journal of Mathematic 


\section{SIMPLE MODULES AND HEREDITARY RINGS}

\section{ABRAHAM ZAKS}

The purpose of this note is to prove that if in a semiprimary ring $A$, every simple module that is not a projective $\Lambda$-module is an injective $\Lambda$-module, then $\Lambda$ is a semi-primary hereditary ring with radical of square zero. In particular, if $A$ is a commutative ring, then $A$ is a finite direct sum of fields. If $\Lambda$ is a commutative Noetherian ring then if every simple module that is not a projective module, is an injective module, then for every maximal ideal $M$ in $\Lambda$ we obtain $\operatorname{Ext}^{1}(\Lambda / M, \Lambda / M)=0$. The technique of localization now implies that $\operatorname{gl} \operatorname{dim} \Lambda=0$.

1. We say that $\Lambda$ is a semi-primary ring if its Jacobson radical $N$ is a nilpotent ideal, and $\Gamma=\Lambda / N$ is a semi-simple Artinian ring.

Throughout this note all modules (ideals) are presumed to be left modules (ideals) unless otherwise stated. For any idempotent $e$ in $\Lambda$ we denote by $N e$ the ideal $N \cap \Lambda e$.

We discuss first semi-primary rings $\Lambda$ with radical $N$ of square zero for which every simple module that is not a projective module is an injective module. We shall study the nonsemi-simple case, i.e., $N \neq 0$.

Under this assumption $N$ becomes naturally a $\Gamma$-module.

Let $e, e^{\prime}$ be primitive idempotents in $\Lambda$ for which $e N e^{\prime} \neq 0$. In particular $N e^{\prime} \neq 0^{\circ}$ From the exact sequence $0 \rightarrow N e^{\prime} \rightarrow \Lambda e^{\prime} \rightarrow S^{\prime} \rightarrow 0$, it follows that $S^{\prime}$ is not a projective module since $\Lambda e^{\prime}$ is indecomposable. Since $S^{\prime}$ is a simple module it follows that $S^{\prime}$ is an injective module.

Next consider the simple module $\Lambda e / N e=S$. Since $e N e^{\prime} \neq 0$, since $N e^{\prime}$ is a $\Gamma$-module, and since on $N$ the $\Gamma$-module structure and the $\Lambda$-module structure coincide, $N e^{\prime}$ contains a direct summand isomorphic with $S$. This gives rise to an exact sequence $0 \rightarrow S \rightarrow \Lambda e^{\prime} \rightarrow K \rightarrow 0$ with $K \neq 0$. If $S$ were injective this sequence would split, and this contradicts the indecomposability of $\Lambda e^{\prime}$. Therefore $S$ is a projective module.

Hence $N e^{\prime}$ is a direct sum of projective modules, therefore $N e^{\prime}$ is a projective module. The exact sequence $0 \rightarrow N e^{\prime} \rightarrow \Lambda e^{\prime} \rightarrow S^{\prime} \rightarrow 0$ now implies $l . p . \operatorname{dim} S^{\prime} \leqq 1$, and since $S^{\prime}$ is not a projective module, then l.p.dim $S^{\prime}=1$.

Hence l.p. $\operatorname{dim}_{\Lambda} \Gamma=1$, and this implies that $\Lambda$ is an hereditary ring (i.e., l.gl.dim $\Lambda=1$ ) [1].

Conversely, assume that $l . g \operatorname{lodim} \Lambda=1$. Every ideal in $\Lambda$ is the direct sum of $N_{1}, \cdots, N_{t}$ where $N_{1}$ is contained in the radical, and 
the others (if any) are components of $\Lambda$, i.e., $N_{i}=\Lambda e_{i}$ where $e_{2}, \cdots, e_{t}$ are primitive orthogonal idempotents in $\Lambda$ [4].

Let $\Gamma e^{\prime}$ be any simple $\Lambda$-module. Since $N_{1} \subset N, N_{1}$ is a $\Gamma$-module. Since on $N$ the $\Gamma$-module structure coincides with the $\Lambda$-module structure, it easily follows that there exists a nonzero map of $N_{1}$ onto $\Gamma e^{\prime}$ if and only if $\Gamma e^{\prime}$ (up to isomorphism) is a direct summand of $N_{1}$. This in particular implies that $\Gamma e^{\prime}$ is a projective $\Lambda$-module, since then $\Gamma e^{\prime}$ is isomorphic to an ideal. If $\Gamma e^{\prime}$ is not a projective $\Lambda$-module, it follows that $\operatorname{Hom}_{1}\left(N_{1}, \Gamma e^{\prime}\right)=0$. As a consequence, every map from an ideal in $\Lambda$ into $\Gamma e^{\prime}$, extends to a map of $\Lambda$ into $\Gamma e^{\prime}$, hence $\Gamma e^{\prime}$ is an injective $\Lambda$-module.

This proves:

THEOREM A. Let $\Lambda$ be a semi-primary ring with radical of square zero. Then every simple A-module that is not a projective A-module is an injective $\Lambda$-module if and only if $\Lambda$ is a hereditary ring.

If $\Lambda$ is a semi-primary ring with radical $N$ and $N^{2} \neq 0$, then a simple module is projective if and only if it is isomorphic to a component, hence if $\Lambda e / N e$ is a projective module $N e=0$, and the idempotent $e$, when reduced $\bmod N^{2}$ (i.e., in $\Lambda / N^{2}$ ) will still give rise to a projective module. If $\Lambda e / N e$ is an injective module $e$ will give rise to an injective $\Lambda / N^{2}$-module. This will follow from the following two lemmas:

Lemma 1. Let $e, e^{\prime}$ be primitive idempotents in 1 . Then $1 e$ is isomorphic to $\Lambda e^{\prime}$ if and only if $\operatorname{Hom}_{\Lambda}\left(\Lambda e^{\prime}, \Lambda e / N e\right) \neq 0$.

Proof. If $\Lambda e$ is isomorphic to $A e^{\prime}$ then obviously

$$
\operatorname{Hom}_{\Lambda}\left(\Lambda e^{\prime}, \Lambda e / N e\right) \neq 0 \text {. }
$$

Conversely, let $f: \Lambda e^{\prime} \rightarrow \Lambda e / N e$ be a nonzero map. Since $\Lambda e / N e$ is a simple module $f$ is an epimorphism. Denote by $\pi$ the canonical projection $\pi: \Lambda e \rightarrow \Lambda e / N e$ then since $\Lambda e^{\prime}$ is a projective module there exists a map $g: \Lambda e^{\prime} \rightarrow \Lambda e$ such that $f=\pi \circ g$. Since $\pi(N e)=0$, it follows that $g$ is an epimorphism. Since $\Lambda e$ is a projective module and $\Lambda e^{\prime}$ an indecomposable module $g$ is an isomorphism.

LEMma 2. Let $S$ be an injective simple 1 -module and $I$ an ideal that is contained in the radical. Then $\operatorname{Hom}_{A}(I, S)=0$.

Proof. Let $f$ be a nonzero map of $I$ into $S$. Since $S$ is an 
injective $\Lambda$ module it follows that $f$ extends to a map of $\Lambda$ onto $S$, $f: \Lambda \rightarrow S$, but this implies that $f(N)=0$. Since $f(I) \subset f(N)$ this is a contradiction. Therefore every map of $I$ into $S$ is the zero map.

THeOREM B. Let $\Lambda$ be a semi-primary ring then the following are equivalent:

(i) $\Lambda$ is an hereditary ring with radical of square zero.

(ii) Every simple module that is not a projective A-module is an injective 1 -module.

Proof. That (i) implies (ii) follows from Theorem A.

(ii) $\Rightarrow$ (i): Let $e_{1}, \cdots, e_{t}$ be a complete set of orthogonal idempotents, i.e., each $e_{i}$ is a primitive idempotent, and

$$
\Lambda=\Lambda e_{1}+\cdots+\Lambda e_{t} \text {. }
$$

Set $S_{i}=\Lambda e_{i} / N e_{i}$. We denote by $\bar{e}_{1}, \cdots, \bar{e}_{t}$ the images of $e_{1}, \cdots, e_{t}$ in $\Lambda / N^{2}$ under the canonical epimorphism $\Lambda \rightarrow \Lambda / N^{2}$. Then $S_{1}, \cdots, S_{t}$ may be viewed as simple $\Lambda / N^{2}$-modules, and every simple $\Lambda / N^{2}$-module is necessarily isomorphic with some $S_{i}$. If $S_{j}$ is $A$-projective then $N e_{j}=0$, and necessarily $S_{j}$ is $\Lambda / N^{2}$-projective. If $S_{j}$ is $\Lambda$-injective then we claim that $S_{j}$ is $\Lambda / N^{2}$-injective. It suffices to prove that for any ideal $I^{\prime}$ in $\Lambda / N^{2}$, and any $\Lambda / N^{2}$-map $f$ from $I^{\prime}$ to $S_{j}, f$ extends to a map of $\Lambda / N^{2}$ into $S_{j}$. Since $I^{\prime}$ is a direct sum of ideals $I_{1}, \cdots, I_{r}^{\prime}$, $I_{1}^{\prime} \subset N / N^{2}$ and the others (if any) are components of $\Lambda / N^{2}$, we will be done if we prove that $\operatorname{Hom}_{A / N^{2}}\left(I^{\prime \prime}, S_{j}\right)=0$ whenever $I^{\prime \prime} \subset N / N^{2}$. Let $I$ be the inverse image of $I^{\prime \prime}$ under the homomorphism $\Lambda \rightarrow \Lambda / N^{2}$, then $\operatorname{Hom}_{1}\left(I, S_{j}\right)=0$ since $I \subset N$ (Lemma 2). If we denote by $h$ the map $I \rightarrow I^{\prime \prime}$ (restriction of the canonical projection) and if $f$ is any map of $I^{\prime \prime}$ into $S_{j}$ then if $f$ is not the zero map, $f \circ h$ from $I$ into $S_{j}$ is a nonzero 1 -map of $I$ into $S_{j}$. This contradiction implies that $S_{j}$ is an injective $\Lambda / N^{2}$-module.

By Theorem $\mathrm{A}$ it now follows, since $\Lambda / N^{2}$ is a semi-primary ring with radical of square zero, that $l \cdot g l \cdot \operatorname{dim} \Lambda / N^{2} \leqq 1$. This necessarily implies that $N^{2}=0$ [2].

Remark that if all simple modules are projective modules, or if all simple modules are injective modules, then $\Lambda$ is a semi-simple ring [1].

Finally, if $N \neq 0$ then there exist a simple projective (injective) module that is not an injective (projective) module. 


\section{REFERENCES}

1. M. Auslander, Global dimension, Nagoya Math. J. 9 (1955), 67-77.

2. S. Eilenberg and T. Nakayama, Dimension of residue rings, Nagoya Math. J. 11 (1957), 9-12.

3. B. L. Osofsky, Rings all of whose finitely generated modules are injective, Pacific J. Math. 14 (1964), 645-650.

4. A. Zaks, Global dimension of Artinian rings, Proc. Amer. Math. Soc. 18 (1967), 1102-1106.

Received December 5, 1967.

Technion, Haifa

ISRAEL 


\section{PACIFIC JOURNAL OF MATHEMATICS}

\section{EDITORS}

\section{H. ROYDEN}

Stanford University

Stanford, California

R. R. Phelps

University of Washington

Seattle, Washington 98105

\section{J. DugundJI}

Department of Mathematics

University of Southern California

Los Angeles, California 90007

RICHARD ARENS

University of California

Los Angeles, California 90024

\section{ASSOCIATE EDITORS}
E. F. BeCKENBACH
B. H. NeumanN
F. WOLF
K. YosIDA

\section{SUPPORTING INSTITUTIONS}

\author{
UNIVERSITY OF BRITISH COLUMBIA \\ CALIFORNIA INSTITUTE OF TECHNOLOGY \\ UNIVERSITY OF CALIFORNIA \\ MONTANA STATE UNIVERSITY \\ UNIVERSITY OF NEVADA \\ NEW MEXICO STATE UNIVERSITY \\ OREGON STATE UNIVERSITY \\ UNIVERSITY OF OREGON \\ OSAKA UNIVERSITY \\ UNIVERSITY OF SOUTHERN CALIFORNIA
}

\author{
STANFORD UNIVERSITY \\ UNIVERSITY OF TOKYO \\ UNIVERSITY OF UTAH \\ WASHINGTON STATE UNIVERSITY \\ UNIVERSITY OF WASHINGTON \\ AMERICAN MATHEMATICAL SOCIETY \\ CHEVRON RESEARCH CORPORATION \\ TRW SYSTEMS \\ NAVAL WEAPONS CENTER
}

Mathematical papers intended for publication in the Pacific Journal of Mathematics should be in typed form or offset-reproduced, double spaced with large margins. Underline Greek letters in red, German in green, and script in blue. The first paragraph or two must be capable of being used separately as a synopsis of the entire paper. It should not contain references to the bibliography. Manuscripts, in duplicate if possible, may be sent to any one of the four editors. All other communications to the editors should be addressed to the managing editor, Richard Arens, University of California, Los Angeles, California 90024.

Each author of each article receives 50 reprints free of charge; additional copies may be obtained at cost in multiples of 50 .

The Pacific Journal of Mathematics is published monthly. Effective with Volume 16 the price per volume (3 numbers) is $\$ 8.00$; single issues, $\$ 3.00$. Special price for current issues to individual faculty members of supporting institutions and to individual members of the American Mathematical Society: $\$ 4.00$ per volume; single issues $\$ 1.50$. Back numbers are available.

Subscriptions, orders for back numbers, and changes of address should be sent to Pacific Journal of Mathematics, 103 Highland Boulevard, Berkeley 8, California.

Printed at Kokusai Bunken Insatsusha (International Academic Printing Co., Ltd.), 7-17, Fujimi 2-chome, Chiyoda-ku, Tokyo, Japan.

PUBLISHED BY PACIFIC JOURNAL OF MATHEMATICS, A NON-PROFIT CORPORATION

The Supporting Institutions listed above contribute to the cost of publication of this Journal, but they are not owners of publishers and have no responsibility for its content or policies. 


\section{Pacific Journal of Mathematics}

\section{Vol. 26, No. $3 \quad$ BadMonth, 1968}

Leonard Asimow, Universally well-capped cones ................. 421

Lawrence Peter Belluce, William A. Kirk and Eugene Francis Steiner,

Normal structure in Banach spaces ..................... 433

William Jay Davis, Bases in Hilbert space.................... 441

Larry Lee Dornhoff, p-automorphic p-groups and homogeneous

algebras..................................... 447

William Grady Dotson, Jr. and W. R. Mann, A generalized corollary of the

Browder-Kirk fixed point theorem ....................... 455

John Brady Garnett, On a theorem of Mergelyan ................. 461

Matthew Gould, Multiplicity type and subalgebra structure in universal

algebras............................................. 469

Marvin D. Green, A locally convex topology on a preordered space . . . . . 487

Pierre A. Grillet and Mario Petrich, Ideal extensions of semigroups . . . . . . 493

Kyong Taik Hahn, A remark on integral functions of several complex

variables ... . . . . . . . . . . . . . . . . . . . . . . . . . . . . . . . . . . . 509

Choo Whan Kim, Uniform approximation of doubly stochastic

operators..................................... 515

Charles Alan McCarthy and L. Tzafriri, Projections in $\mathscr{L}_{1}$ and

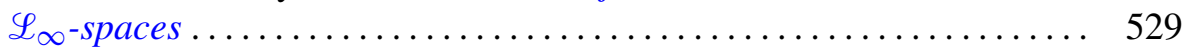

Alfred Berry Manaster, Full co-ordinals of RETs ................ 547

Donald Steven Passman, $p$-solvable doubly transitive permutation

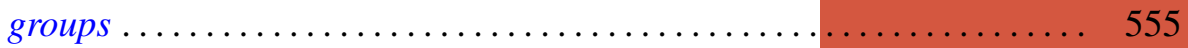

Neal Jules Rothman, An $L^{1}$ algebra for linearly quasi-ordered compact

semigroups ....................................... 579

James DeWitt Stein, Homomorphisms of semi-simple algebras .......... 589

Jacques Tits and Lucien Waelbroeck, The integration of a Lie algebra

representation ...............................

David Vere-Jones, Ergodic properties of nonnegative matrices. II ........ 601

Donald Rayl Wilken, The support of representing measures for $R(X) \ldots \ldots 621$

Abraham Zaks, Simple modules and hereditary rings .... . . 